\title{
Thalamic changes in a preterm sample with periventricular leukomalacia: correlation with white-matter integrity and cognitive outcome at school age
}

\author{
Leire Zubiaurre-Elorza', Sara Soria-Pastor², Carme Junqué', Davinia Fernandez-Espejo', Dolors Segarra', Núria Bargalló3, \\ Camila Romano-Berindoague ${ }^{4}$ and Alfons Macaya ${ }^{4}$
}

INTRODUCTION: Thalamic abnormalities have been well documented in preterms with periventricular leukomalacia (PVL), although their contribution to long-term cognitive dysfunctions has not been thoroughly investigated.

RESULTS: Significant differences between groups were observed for global thalamic volume. Neuropsychological assessments showed that preterms with PVL scored within the normal range, although significantly below controls in the full intelligence quotient and the specific cognitive domains of processing speed and working memory. Correlations of several thalamic regions with Working Memory Index and FIQ were found in the PVL group. Moreover, thalamic atrophy correlated with white-matter (WM) damage indexes (fractional anisotropy and radial diffusivity) assessed by diffusion tensor imaging.

DISCUSSION: The findings suggest that thalamic damage is a common correlate of WM microstructural alterations and might be involved in the cognitive deficits seen in premature infants with PVL at school age.

METHODS: We analyzed the impact of PVL-associated thalamic injury on cognitive status at school age and its correlation with WM integrity as measured by magnetic resonance imaging techniques. Thalamic volume and shape of 21 preterm children with PVL were compared with those of 11 preterm children of similar gestational age and birth weight with no evidence of focal WM abnormality.

$\mathbf{P}$ eriventricular leukomalacia (PVL) is an injury affecting cerebral white matter (WM), commonly associated with preterm birth. A recent review concluded that PVL WM injury is often accompanied by diffuse neuronal/axonal disease affecting cortical and subcortical gray matter (GM) (1).

The impact of cerebral impairment in preterm infants is well documented. However, quantitative evidence to substantiate whether deep GM abnormalities in preterm patients with PVL are related to cognitive problems in childhood is lacking. A neuropathological study showed neuronal loss, greater in thalamus than in cerebral cortex, in subjects with PVL (2), and magnetic resonance imaging (MRI) studies have shown impairment in cortical and subcortical GM structures (3-5). The main subcortical structure that has been studied and found reduced in chronic PVL is the thalamus (6-8), its dorsomedial and pulvinar regions being reported as the more often affected areas (8-10).

Diffusion tensor imaging (DTI) is an MRI technique sensitive to water diffusion that allows quantitation of different indexes of WM integrity: fractional anisotropy (FA, degree of directionality within the fibers), mean diffusivity (MD, average of water diffusion), radial diffusivity (RD, perpendicular diffusivity), and axial diffusivity ( $\mathrm{AD}$, parallel diffusivity). As a consequence of normal brain maturation, almost all WM regions show a pattern of FA increments as well as MD decrements occurring from early childhood to adolescence (11). However, studies in children with PVL have evidenced an inverse pattern of diffusion in some brain areas (decrements in FA and increments in $\mathrm{MD}$ ) as compared with controls $(12,13)$. A high FA index reflects preserved WM, whereas a high MD index indicates tissue alteration (14). DTI has been applied as a sensitive technique for detection of damage to specific WM tracts in patients with PVL $(15,16)$, and a recent study has related thalamic injury, specifically pulvinar involvement, to the parieto-occipital WM implicated in the cognitive visual network (10).

Given the relevance of the thalamus to complex cognitive functions and its well-established connectivity with high-order neocortical regions, we hypothesized that (i) preterm children with PVL would show abnormalities in thalamic structure as compared with preterm children with no evidence of focal WM abnormality, (ii) these abnormalities could explain deficits in cognitive performance, and (iii) WM impairment would correlate with thalamic volume reductions. To our knowledge, this is the first study to directly correlate thalamic volume and cognitive functions in patients with PVL.

\section{RESULTS}

Clinical Data Base and Cognitive Results

Comparisons between groups regarding neonatal and demographic data are detailed in Table 1 . The groups did not differ with regard to gestational age (GA), birth weight, birth length,

'Department of Psychiatry and Clinical Psychobiology, Faculty of Medicine, University of Barcelona, Barcelona, Spain; ${ }^{2}$ Clinical Institute of Neurosciences, Hospital Clínic Barcelona, Barcelona, Spain; ${ }^{3}$ Neuroradiology Section, Radiology Department, Centre de Diagnòstic per la Imatge Clínic, Hospital Clínic, Barcelona, Spain; ${ }^{4}$ Secció de Neurologia Infantil, Vall d'Hebron University Hospital, Autonomous University of Barcelona, Barcelona, Spain. Correspondence: Carme Junqué (cjunque@ub.edu) 
head circumference, age at scan, gender, laterality, or neonatal clinical data, excepting the greater incidence of mild intraventricular hemorrhage (IVH) in the PVL group. Neuromotor sequelae were present in eight patients, six of them displaying mild hand clumsiness (Table 2).

Neuropsychological performance is detailed in Table 3. Although within the normal range for cognitive performance,

Table 1. Clinical characteristics of study children

\begin{tabular}{|c|c|c|c|}
\hline & $\begin{array}{c}\text { PVL group } \\
\text { (mean } \pm S D, \\
n=21)\end{array}$ & $\begin{array}{l}\text { Non-PVL group } \\
\text { (mean } \pm \text { SD } \\
n=11)\end{array}$ & $\begin{array}{l}\text { Statistics } \\
\text { ( } P \text { value })\end{array}$ \\
\hline \multicolumn{4}{|l|}{ Neonatal data } \\
\hline Gestational age, wk & $30.24 \pm 2.99$ & $31.45 \pm 0.82$ & $\begin{array}{c}t=-1.74 \\
(.094)\end{array}$ \\
\hline Birth weight, $g$ & $1547 \pm 552$ & $1597 \pm 484$ & $\begin{array}{c}t=-0.25 \\
(.802)\end{array}$ \\
\hline Length, $\mathrm{cm}^{\mathrm{a}}$ & $39.54 \pm 3.94$ & $41.36 \pm 4.66$ & $\begin{array}{c}t=-1.02 \\
(.321)\end{array}$ \\
\hline Head circumference, $\mathrm{cm}^{\mathrm{a}}$ & $27.42 \pm 2.71$ & $29.23 \pm 2.65$ & $\begin{array}{c}t=-1.62 \\
(.121)\end{array}$ \\
\hline \multicolumn{4}{|l|}{ Demographic data } \\
\hline Gender, M/F & $12 / 9$ & $7 / 4$ & $\begin{array}{c}X^{2}=0.13 \\
(.722)\end{array}$ \\
\hline Age at scan, y (range) & $\begin{array}{c}8.71 \pm 1.79 \\
(6-12)\end{array}$ & $\begin{array}{c}9.00 \pm 0.63 \\
(8-10)\end{array}$ & $\begin{array}{c}t=-0.66 \\
(.517)\end{array}$ \\
\hline Right-handed, $n$ (\%) & $16(76)$ & $10(91)$ & $\begin{array}{c}X^{2}=1.03 \\
(.311)\end{array}$ \\
\hline Parental education, $n(\%)$ & & & $\begin{array}{c}X^{2}=2.02 \\
(.365)\end{array}$ \\
\hline High & $6(29)$ & $5(46)$ & \\
\hline Intermediate & $6(29)$ & $4(36)$ & \\
\hline Low & $9(42)$ & $2(18)$ & \\
\hline
\end{tabular}

F, female; M, male; PVL, periventricular leukomalacia.

aNeonatal length and head circumference available for $n=12$ in the preterm with PVL group. preterm children with PVL scored below their preterm peers. No differences were found when comparing preterms with PVL and IVH vs. those without IVH for all the variables analyzed.

\section{Clinical MRI Findings}

Current MRI studies were consistent with the neuroradiological diagnosis of PVL in all cases, except for one who showed no signs of brain damage and was excluded. On the basis of magnitude of WM reduction and gliosis, patients were classified as having mild $(n=9)$, moderate $(n=7)$, or severe $(n=5) \mathrm{PVL}$. In this small sample, no comparison between these subgroups was attempted. MRI studies in preterm controls showed no evidence of brain abnormalities at school age.

\section{Volumetric Results}

Differences between groups in bilateral thalamic volumetric measures (left: $F_{1,28}=11.19 ; P=.002$; right: $F_{1,28}=7.93 P=.009$ ) and normalized brain parenchymal volume (NBPV) $\left(F_{1,28}=\right.$ 6.40; $P=.017)$ showed significantly lower values in preterm children with PVL. Comparisons between groups regarding thalamic shape analysis did not show statistically significant differences in any specific region of the thalamus.

Left thalamic volume correlated with Working Memory Index and full intelligence quotient (FIQ) in children with PVL (Table 4). Mean FA, in turn, did not correlate with cognitive data (data not shown). Shape analysis evidenced a statistically significant correlation between some specific regions of the thalamus bilaterally (anterior, dorsomedial, and pulvinar) and FIQ and Working Memory Index (Figure 1 and Table 5). No significant correlations were found between thalamic structure (volume or shape) and cognitive variables for children with no evidence of focal WM abnormality (control group).

Correlation analyses with WM integrity showed that thalamic volume positively correlated with FA and negatively with $\mathrm{RD}$ index (Figure 2). The pattern of correlations involved the posterior cerebral regions with several associative WM fibers,

Table 2. Clinical data of preterm children

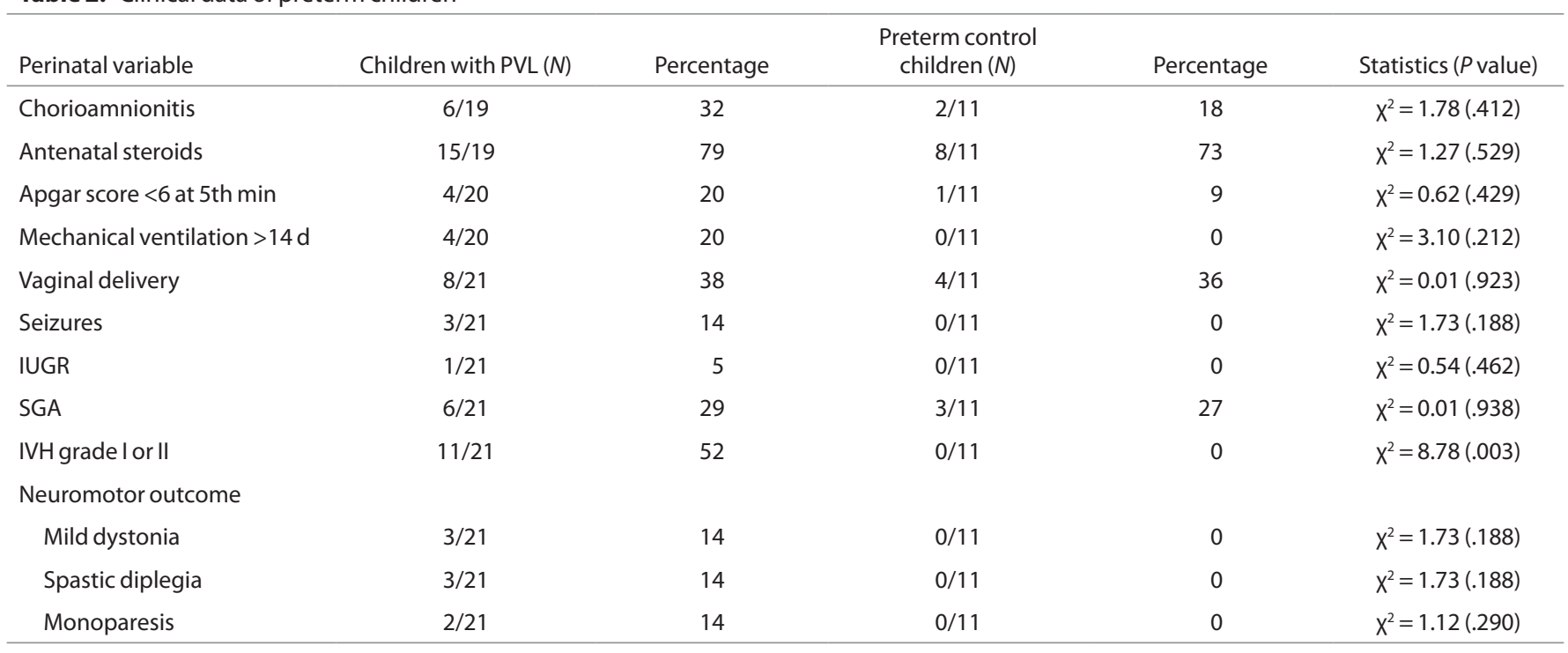

IUGR, intrauterine growth restriction; IVH, intraventricular hemorrhage; PVL, periventricular leukomalacia; SGA, small for gestational age. 
including inferior longitudinal, superior longitudinal, and inferior fronto-occipital fasciculi; the cingulum, and posterior commissural fibers (splenium of the corpus callosum). Integrity of some projection fibers, such as anterior thalamic radiation and corticospinal tract, also correlated with thalamic volume.

\section{DISCUSSION}

The aims of this quantitative MRI study were to analyze global and local thalamic structures and to investigate their correlation with cognitive performance and their relationship

Table 3. Neuropsychological data

\begin{tabular}{lccc}
\hline $\begin{array}{l}\text { Neuropsychological } \\
\text { data }\end{array}$ & $\begin{array}{c}\text { PVL group } \\
(\text { mean } \pm \text { SD, } \\
n=21)\end{array}$ & $\begin{array}{c}\text { Non-PVL group } \\
(\text { mean } \pm \text { SD, } \\
n=11)\end{array}$ & $\begin{array}{c}\text { Statistics } \\
(P \text { value })\end{array}$ \\
\hline $\begin{array}{l}\text { WISC-IV } \\
\quad \text { Verbal }\end{array}$ & $101.62 \pm 12.60$ & $108.73 \pm 15.01$ & $\begin{array}{c}t=-1.42 \\
\text { Comprehension } \\
\text { Index }\end{array}$ \\
$\begin{array}{l}\text { Perceptual } \\
\text { Reasoning Index }\end{array}$ & $93.62 \pm 14.54$ & $98.09 \pm 13.79$ & $t=-0.84$ \\
Working Memory & $90.57 \pm 17.52$ & $105.45 \pm 17.08$ & $\begin{array}{c}(.407) \\
\text { Index }\end{array}$ \\
$\begin{array}{l}\text { Processing Speed } \\
\text { Index }\end{array}$ & $92.76 \pm 15.43$ & $106.36 \pm 8.79$ & $\begin{array}{c}(.028) \\
t=-3.17 \\
\end{array}$ \\
& & & $(.003)$ \\
FIQ & $92.48 \pm 14.56$ & $104.36 \pm 13.63$ & $t=-2.24$ \\
\end{tabular}

FIQ, full intelligence quotient; PVL, periventricular leukomalacia; WISC-IV, Wechsler Intelligence Scales for Children-Version IV.

Table 4. Correlational analysis between thalamic volume and cognitive variables

\begin{tabular}{lcc}
\hline Thalamic volume & Statistics $(P$ value $)$ & Statistics $(P$ value $)$ \\
\hline Cognitive variables & Left hemisphere & Right hemisphere \\
Verbal Comprehension Index & $r=0.23(.351)$ & $r=0.09(.715)$ \\
Perceptual Reasoning Index & $r=0.41(.080)$ & $r=0.39(.095)$ \\
Working Memory Index & $r=0.47(.043)$ & $r=0.31(.193)$ \\
Processing Speed Index & $r=0.43(.068)$ & $r=0.37(.117)$ \\
FIQ & $r=0.50(.028)$ & $r=0.39(.099)$ \\
\hline
\end{tabular}

FIQ, full intelligence quotient. with WM integrity in a sample of school-aged children born preterm with PVL. Our results indicate that cases with PVL showed overall volumetric reduction of the thalamus as compared with a preterm control group with no evidence of WM focal abnormality. Correlation analyses in the preterm PVL group showed that thalamic reductions and specifically anterior, dorsomedial, and pulvinar regions have an effect on general intelligence and working memory measures. In addition, the results quantitatively evidenced a clear relationship of thalamic atrophy with the presence of posterior WM injury. To our knowledge, this is the first quantitative MRI report on a PVL sample where alterations in thalamic volume or shape are linked to FIQ and WM microstructure.

Our preterm children with PVL showed diminished global thalamic volume as compared with children with no evidence of focal WM abnormality. Volume decreases in subcortical GM structures have been previously reported in preterm cohorts' studies without specific consideration of cases with PVL. These MRI studies performed in heterogeneous groups of preterm children used different quantitative methodologies and reported cortical and subcortical GM volume decreases in infancy $(1,17,18)$, childhood $(19,20)$, or adolescence $(21)$. Thalamic damage is also a common finding in PVL neuropathological studies $(2,8)$, as well as MRI studies $(6,7)$ and PVL mouse models (22). Moreover, quantitation of neuronal loss in a PVL postmortem study showed that the most affected GM structure was the thalamus, with a $38 \%$ loss that surpassed the $21 \%$ found in the cerebral cortex (2). Due to the wide age range of the subjects included in this study, we controlled all our analyses by age at scan, but it is worth noting that we did not take into account the potential influence of pubertal stage. However, in a study, the thalamus was not among the brain regions displaying enhanced sexual brain dimorphism during puberty (23).

The relationship between cerebral damage and cognitive deficits in preterm infants has been extensively investigated form birth to adulthood. It is known that in preterms, WM injury does appear in association with GM abnormalities (24) and that cortical and subcortical GM impairment is likely to influence cognitive performance. Previous quantitative MRI studies in preterm children found that regional GM changes in regions such as sensorimotor, middle temporal, and postcentral gyri correlated with

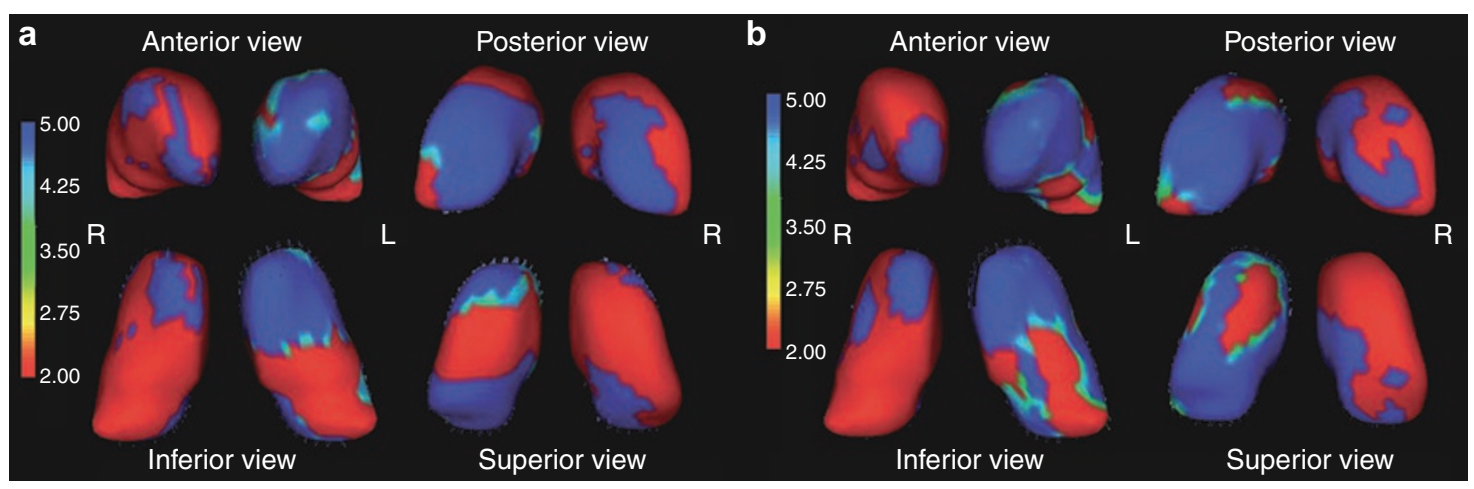

Figure 1. Shape correlation analysis between thalamic regions: (a) full intelligence quotient and (b) Working Memory Index in the PVL group at childhood. L, left; R, right. Regions achieving significance are represented in blue color. PVL, periventricular leukomalacia. 
FIQ $(19,20)$. It has also been reported that frontal and temporal volume decreases correlate with verbal intelligence quotient decline in preterm children, whereas decreases in temporal and occipital cortical regions are associated with a decline in the

Table 5. Correlational analysis between thalamic structure (shape or volume) and cognitive variables or DTI indexes

\begin{tabular}{ll}
\hline Thalamic structure $^{\mathrm{a}}$ & \multicolumn{1}{c}{ Statistics ( $P$ value) } \\
\hline $\begin{array}{l}\text { Thalamic shape and cognitive variables } \\
\text { Working Memory Index }\end{array}$ & \\
& Left: $F_{3,15}=3.62(.038)$ \\
Right: $F_{3,15}=5.35(.010)$ \\
LIQ & Left: $F_{3,15}=3.86(.031)$ \\
Right: $F_{3,15}=5.24(.011)$ \\
FA & \\
RD & $r=0.73(<.001)$ \\
\hline
\end{tabular}

DTI, diffusion tensor imaging; FA, fractional anisotropy; FIQ, full intelligence quotient; NBPV, normalized brain parenchymal volume; RD, radial diffusivity.

aThe analyses are controlled by age at evaluation and NBPV. performance intelligence quotient (25). In a study of normal brain development during childhood, both cortical and subcortical GM volumes correlated with FIQ, although cortical GM shows the strongest correlations (26). Given the evidence of impaired thalamocortical connections in preterms with WM injury (27), thalamic damage, as our results support, might play a role in the low performance on the intelligence test. However, we acknowledge the possible influence on cognition of other WM and cortical GM areas in preterm children with evidence of PVL.

Studies in premature children with WM injury have found thalamic abnormalities in specific regions such as pulvinar $(9,10)$ and dorsomedial nuclei $(8,28)$, their presence being associated with poorer cognitive outcomes. Correlation analyses in our study demonstrated that FIQ and Working Memory Index correlated with anterior, dorsomedial, and pulvinar areas, in keeping with studies of thalamocortical connectivity describing connections between the human dorsomedial thalamic nucleus and frontal and temporal areas (29), already present in healthy preterms (27). Shape abnormalities in the anterior nuclei, which are connected with the prefrontal cortex, could

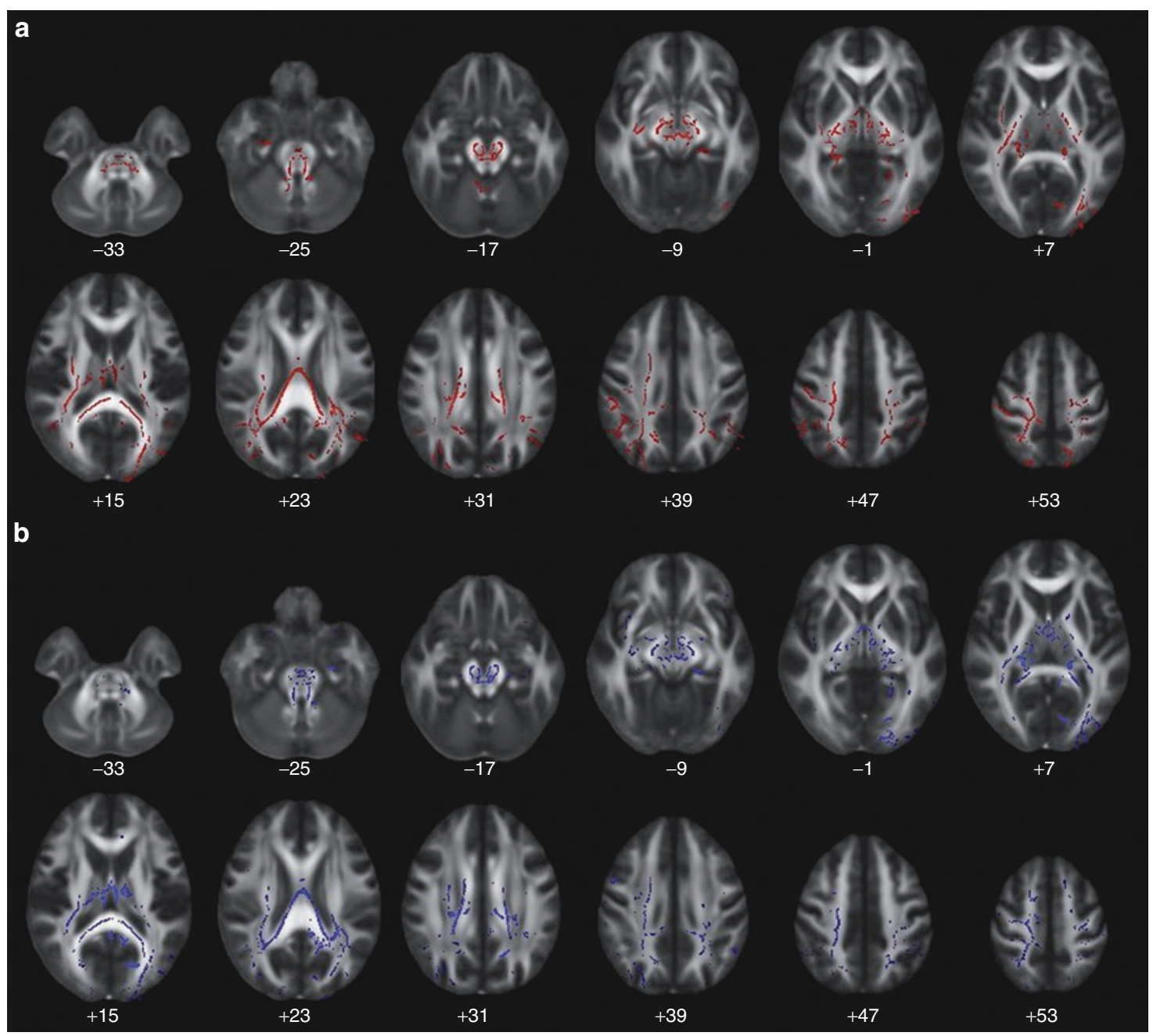

Figure 2. Family-wise error-corrected results ( $P \leq 0.05)$ : (a) positive correlation between thalamic volume and posterior fractional anisotropy displayed in red color and (b) negative correlation between thalamic volume and posterior radial diffusivity is displayed in blue color. Numbers represent the corresponding slice. 
also translate into low working memory performance. The dorsomedial area was found to be involved in working memory deficits (30), and pulvinar abnormalities have been proposed to interfere with cognitive visual performance in preterm children with PVL (10). It is worth noting that the thalamus has structural and functional relationships with the basal ganglia and that the caudate nucleus play an important role in cognitive dysfunctions (31). Moreover, it is known that the latter structure correlated with cognitive scores in preterm samples (32). Further studies should focus on caudate structure and its correlations with cognition in homogeneous PVL samples.

Although the cognitive scores of our preterm sample were within the normal range, children with PVL had lower general intelligence scores, as other preterm studies have reported (33). Our preterms with PVL showed good performance in cognitive tasks because we excluded those with a FIQ $\leq 70$. This was a main exclusion criterion to allow the assessment of specific domains of cognition. Thus, our results cannot be generalized to all preterms with PVL at school age. The greater differences were found in the Processing Speed Index. As well as a measure of attention and quick scanning, the index clearly depends on visuospatial and visuoconstructive functions, reported altered in preterm children with PVL (34). It is conceivable that minor motor impairment could have affected the performance in the Perceptual Reasoning and Processing Speed indexes.

However, the thalamic reduction was related to posterior WM microstructural damage. A positive correlation between thalamic volume and FA in posterior tracts was found; the more affected the thalamus, the more impaired the posterior WM. A causative mechanism cannot be established with certainty and whether thalamic damage in PVL samples is due to a primary injury, maturational/trophic disturbances, or both, is still unresolved (1). Parieto-occipital WM impairment is one of the characteristic findings of preterms with PVL (35), and in a recent ultrasound study, it was related to thalamic damage (36). Our data also revealed that decreased thalamic volume correlated with an increase in RD. Nagasunder et al. (10) found increased thalamic RD in the most severe cases with PVL, arguably due to primary demyelination (37). A distinct feature of our study was selecting a most appropriate control cohort, that of age-matched preterms with normal brain imaging.

To sum up, in this sample of preterm children with PVL, decreased thalamic volume was associated with poorer cognitive performance and posterior WM microstructural abnormalities. Further follow-up studies in a PVL preterm cohort, as the children reach adolescence and young adulthood, will be required to determine the long-lasting cognitive consequences of PVL.

\section{METHODS}

\section{Participants}

Between June 2008 and May 2009, we recruited a sample of children (6-14 y old) born preterm with a history of PVL. These children belong to a cohort followed prospectively at the Hospital Universitari Vall d'Hebron (Barcelona, Spain). Inclusion criteria for the children with PVL were preterm birth with a GA $<37 \mathrm{wk}$ and diagnostic signs of PVL (periventricular echodensities or cysts, corresponding to Grade II-IV
PVL) on neonatal cranial ultrasound. The diagnosis of PVL was corroborated by current MRI, based on the criteria proposed by Flodmark et al. (38) as adapted and used in a previous quantitative MRI study (7). Exclusion criteria for the PVL group were concurrence of neonatal peri-intraventricular hemorrhage Grade III/IV or any other perinatal brain injury. The initial sample comprised 75 neonates born between 1994 and 2003 with PVL. Whole sample exclusion criteria were any contraindications for MRI, history of acquired brain injury, and a FIQ of 70 or less. Eleven children were excluded on the basis of GA $>37 \mathrm{wk}$, two had FIQ $<70$, one had no evidence of PVL in the current MRI, one had motion artifact in the DTI images $(n=1), 21$ were lost to follow-up, and 18 refused to participate or to undergo the MRI study, which left 21 children who were included in the PVL patient group.

The control group was composed of 11 children born preterm with normal brain imaging, selected from a cohort enrolled in previous studies $(19,39)$ and followed at the Pediatrics Service of the Hospital Clinic (Barcelona, Spain). This was considered the most appropriate control group as they were also born prematurely but without any focal brain injury and did not differ from preterms with PVL in GA, birth weight, age at evaluation (range 8-10 y), or gender distribution. A parental education variable was included representing the highest educational level achieved by any of the parents.

All the participants were subjected to a MRI study and a cognitive assessment by means of the Wechsler Intelligence Scales for ChildrenVersion IV (40) at the Institut d'Investigacions Biomèdiques August Pi i Sunyer (IDIBAPS) Research Institute (Barcelona, Spain). This test provides four separate indexes (Verbal Comprehension, Perceptual Reasoning, Working Memory, and Processing Speed) whose sum provides the FIQ score. All parents provided prior written informed consent and the Ethics Committee of the University of Barcelona approved the study.

\section{MRI Acquisition}

All participants were imaged unsedated at the Centre de Diagnòstic per la Imatge (Hospital Clínic, Barcelona, Spain). MRI images were performed on a TIM TRIO 3-Tesla machine (Siemens, Erlangen, Germany). T1-weighted image acquisition parameters were the same for all subjects: a magnetization prepared rapid acquisition gradient echo sequence in sagittal orientation $(\mathrm{TR} / \mathrm{TE}=2,300 / 2.98 \mathrm{~ms}$; TI $=$ $900 \mathrm{~ms} ; 256 \times 256$ matrix, flip angle $9^{\circ}, 1 \mathrm{~mm}^{3}$ isotropic voxel). DTI was acquired in 30 noncollinear directions using the following parameters: TR/TE $=8,400 / 90$, slice thickness $2 \mathrm{~mm}$, voxel size $2 \times 2 \times 2 \mathrm{~mm}$, $b$ value $1,000 \mathrm{~s} / \mathrm{mm}^{2}$. Two repetitions of this sequence were performed, and the scanning time for each DTI acquisition was 4:47 min.

To detect any lesion (not noticeable on the T1-weighted images) in the PVL preterm group, we also acquired T2-weighted images in axial orientation $\left(\mathrm{TR} / \mathrm{TE}=5,150 / 99 \mathrm{~ms} ; 512 \times 307\right.$ matrix, flip angle $120^{\circ}$, slice thickness $5 \mathrm{~mm}$ with a 1.5 intersection gap) and fluid-attenuated inversion recovery images (axial orientation, TR/TE $=9,040 / 85 \mathrm{~ms}$; TI $=2,500 ; 256 \times 171$ acquisition matrix, flip angle $150^{\circ}$, slice thickness $5 \mathrm{~mm}$ with a 1.5 intersection gap). For children born preterm without PVL, T2-weighted images (axial orientation, TR/TE $=5,533 / 88 \mathrm{~ms}$, $122 \times 122$ matrix, flip angle $90^{\circ}$, slice thickness $2 \mathrm{~mm}$, gap $=0.6 \mathrm{~mm}$ ) were also obtained. Although the T2 images were acquired using different parameters, these images were used only for clinical evaluation of WM intensities. All MRI scans were reviewed by two clinical neuroradiologists to identify PVL at current age or other brain abnormalities. The specific criteria used to diagnose mild, moderate, or severe PVL in the school-age MRI were based on the extent of WM injury and previously reported (5).

\section{Image Analysis}

T1-weighted images were processed with a part of the Functional MRI of the Brain Software Library (41) toolbox named SIENAX (42). First, brain and skull images were extracted for each subject followed by a registration of the brain image to MNI152 space using the skull image as the scaling determinant. Finally, brain tissues were segmented and normalized for head size; GM and WM volumes and NBPV were obtained.

We applied the FIRST (43) tool from Functional MRI of the Brain Software Library to automatically segment the thalami in our sample 


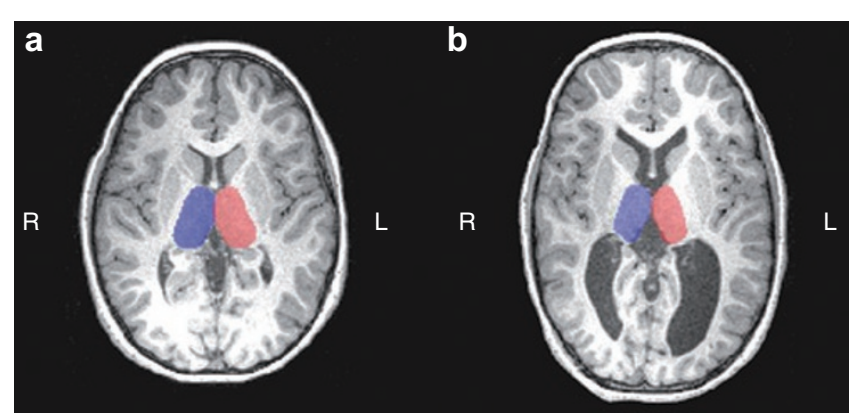

Figure 3. Thalamic segmentation results: (a) children with no evidence of focal white-matter abnormality and (b) a child born preterm with PVL. L, left; $P V L$, periventricular leukomalacia; $\mathrm{R}$, right.

to compare these volumes between groups (Figure 3). It performs two different 12- $d f$ registrations: (i) to MNI152 space, (ii) using an MNI152 subcortical mask to remove voxels that fall outside these structural limits. The shape and appearance models (based on multivariate Gaussian assumptions) used to segment are supplied by Morphometric Analysis (Massachusetts General Hospital, Boston, Massachusetts); their training data include children from age $4 \mathrm{y}$ onwards. Thalamic volumetric data for each child were automatically provided.

Next, a per-vertex shape analysis of the thalamus was performed to determine if any parts of this structure were more affected. This analysis is complementary to the volumetric results and permits identification of local GM changes by examining vertex locations. In the earliest registration of FIRST analysis, a surface mesh containing the vertex location was also created. This mesh compiles a set of triangles and each apex is called a vertex. Then the surface was reconstructed in native space and aligned to the mean surface using 6 degrees of freedom. This surface-based point distribution model allows us the comparison of the vertices between groups as well as correlations with cognitive variables observing the orientation of the vectors (local expansion/contraction) within the structure displayed in a threedimensional rendering (43).

For DTI analyses, eddy current correction was applied, where diffusion weighted images are registered to a reference image $\left(b_{0}\right)$ to remove any image artifact. Nonbrain tissue was removed from each image with the brain extraction tool (42), and FA maps were provided by using DTIFIT, a Functional MRI of the Brain diffusion toolbox.

Tract-based spatial statistics, version 1.2, implemented in Functional MRI of the Brain Software Library (44), was applied to the FA, MD, $\mathrm{AD}(\lambda 1)$, and $\mathrm{RD}(\lambda 2+\lambda 3 / 2)$ maps in our PVL group. Nonlinear registration was performed, the $\mathrm{FA}$, the most representative image of the sample, was selected and affine-aligned to the MNI152 standard space; each FA image was transformed into the standard space by combining the nonlinear transformation to the target FA image with the affine transformation from that target to MNI152 space. A mean FA image was created with all aligned FA images and thinned to produce a skeletonized mean FA image representing the centers of all tracts common to the entire PVL sample. The mean FA skeleton was thresholded to 0.2 to include major WM pathways and to exclude peripheral tracts and GM. Each subject's aligned FA data was then projected onto the skeleton.

\section{Statistical Analysis}

First, all studied variables were tested for homogeneity and normality. The Student's $t$ test was used for quantitative data and the $\chi^{2}$ test of independence for comparing categorical variables. A $P$ value of $\leq .05$ was considered statistically significant. All statistical analyses were carried out with SPSS Version 16.0 (SPSS Inc., Chicago, Illinois).

A univariate analysis of variance to test the differences in NBPV was carried out including age at scan as covariate. Multivariate analysis (Fisher-Snedecor distribution) of covariance was performed to test differences between groups in the bilateral thalamic values with NBPV and age at scan as covariates. For shape analysis of the thalamus correlations with cognitive variables, age at scan and NBPV were added as covariates. For shape analysis, a multivariate $F$ test for each vertex was separately performed by using a Multivariate General Linear Model with Pillai's Trace as the statistical test. All the results underwent an false discovery rate correction method for multiple comparisons. Comparisons between groups and correlations between thalamic vertices and cognitive variables in each group were conducted using $F$ statistics with age at scan and NBPV added as covariates.

Partial correlations were conducted in both preterm groups to test whether the thalamic volume or WM changes in the PVL group (mean FA) correlated with cognitive variables. We did not correct for multiple comparisons given the small sample size and the interdependence of the analyzed cognitive variables $(45,46)$. Due to the substantial proportion of mild neonatal IVH within the PVL group, we tested this variable in all the analyses to determine if it exerted an effect on our results. Voxelwise statistical analyses were carried out in the preterm with PVL group by means of Randomise (47) to assess the relationship between the thalamic volume and the FA, MD, $\mathrm{AD}$, and RD indexes.

\section{ACKNOWLEDGMENTS}

We thank Silvia Juanes for technical support. We give special thanks to the families and children who participated in this study.

\section{STATEMENT OF FINANCIAL SUPPORT}

The authors state that this study was supported by grants from the Ministerio de Ciencia y Tecnología (SAF2005-007340) and the Generalitat de Catalunya (2009 SGR 00941, 2009 SGR 0078). S.S.-P., D.F.-E., and L.Z.-E. hold fellowships from the Ministerio de Educación y Ciencia (AP2005-0047, AP2006-00862, and AP2008-00935, respectively).

\section{REFERENCES}

1. Volpe JJ. Brain injury in premature infants: a complex amalgam of destructive and developmental disturbances. Lancet Neurol 2009;8:110-24.

2. Pierson CR, Folkerth RD, Billiards SS, et al. Gray matter injury associated with periventricular leukomalacia in the premature infant. Acta Neuropathol 2007;114:619-31.

3. Inder TE, Huppi PS, Warfield S, et al. Periventricular white matter injury in the premature infant is followed by reduced cerebral cortical gray matter volume at term. Ann Neurol 1999;46:755-60.

4. Tzarouchi LC, Astrakas LG, Zikou A, et al. Periventricular leukomalacia in preterm children: assessment of grey and white matter and cerebrospinal fluid changes by MRI. Pediatr Radiol 2009;39:1327-32.

5. Zubiaurre-Elorza L, Soria-Pastor S, Junque C, et al. Gray matter volume decrements in preterm children with periventricular leukomalacia. Pediatr Res 2011;69:554-60.

6. Lin Y, Okumura A, Hayakawa F, Kato K, Kuno T, Watanabe K. Quantitative evaluation of thalami and basal ganglia in infants with periventricular leukomalacia. Dev Med Child Neurol 2001;43:481-5.

7. Ricci D, Anker S, Cowan F, et al. Thalamic atrophy in infants with PVL and cerebral visual impairment. Early Hum Dev 2006;82:591-5.

8. Ligam P, Haynes RL, Folkerth RD, et al. Thalamic damage in periventricular leukomalacia: novel pathologic observations relevant to cognitive deficits in survivors of prematurity. Pediatr Res 2009;65:524-9.

9. Yokochi K. Thalamic lesions revealed by MR associated with periventricular leukomalacia and clinical profiles of subjects. Acta Paediatr 1997;86:493-6.

10. Nagasunder AC, Kinney HC, Blüml S, et al. Abnormal microstructure of the atrophic thalamus in preterm survivors with periventricular leukomalacia. AJNR Am J Neuroradiol 2011;32:185-91.

11. Moon WJ, Provenzale JM, Sarikaya B, et al. Diffusion-tensor imaging assessment of white matter maturation in childhood and adolescence. AJR Am J Roentgenol 2011;197:704-12.

12. Thomas B, Eyssen M, Peeters R, et al. Quantitative diffusion tensor imaging in cerebral palsy due to periventricular white matter injury. Brain 2005;128(Pt 11):2562-77. 
13. Fan GG, Yu B, Quan SM, Sun BH, Guo QY. Potential of diffusion tensor MRI in the assessment of periventricular leukomalacia. Clin Radiol 2006;61:358-64.

14. Mori S, Zhang J. Principles of diffusion tensor imaging and its applications to basic neuroscience research. Neuron 2006;51:527-39.

15. Nagae LM, Hoon AH Jr, Stashinko E, et al. Diffusion tensor imaging in children with periventricular leukomalacia: variability of injuries to white matter tracts. AJNR Am J Neuroradiol 2007;28:1213-22.

16. Murakami A, Morimoto M, Yamada K, et al. Fiber-tracking techniques can predict the degree of neurologic impairment for periventricular leukomalacia. Pediatrics 2008;122:500-6.

17. Peterson BS, Anderson AW, Ehrenkranz R, et al. Regional brain volumes and their later neurodevelopmental correlates in term and preterm infants. Pediatrics 2003;111(5 Pt 1):939-48.

18. Boardman JP, Counsell SJ, Rueckert D, et al. Abnormal deep grey matter development following preterm birth detected using deformation-based morphometry. Neuroimage 2006;32:70-8.

19. Soria-Pastor S, Padilla N, Zubiaurre-Elorza L, et al. Decreased regional brain volume and cognitive impairment in preterm children at low risk. Pediatrics 2009;124:e1161-70.

20. Peterson BS, Vohr B, Staib LH, et al. Regional brain volume abnormalities and long-term cognitive outcome in preterm infants. JAMA 2000;284:1939-47.

21. Nosarti C, Giouroukou E, Healy E, et al. Grey and white matter distribution in very preterm adolescents mediates neurodevelopmental outcome. Brain 2008;131(Pt 1):205-17.

22. Shen Y, Plane JM, Deng W. Mouse models of periventricular leukomalacia. J Vis Exp 2010;39:1951.

23. Bramen JE, Hranilovich JA, Dahl RE, et al. Puberty influences medial temporal lobe and cortical gray matter maturation differently in boys than girls matched for sexual maturity. Cereb Cortex 2011;21:636-46.

24. Inder TE, Warfield SK, Wang H, Hüppi PS, Volpe JJ. Abnormal cerebral structure is present at term in premature infants. Pediatrics 2005;115:286-94.

25. Isaacs EB, Edmonds CJ, Chong WK, Lucas A, Morley R, Gadian DG. Brain morphometry and IQ measurements in preterm children. Brain 2004;127(Pt 12):2595-607.

26. Reiss AL, Abrams MT, Singer HS, Ross JL, Denckla MB. Brain development, gender and IQ in children. A volumetric imaging study. Brain 1996;119 (Pt 5):1763-74.

27. Counsell SJ, Dyet LE, Larkman DJ, et al. Thalamo-cortical connectivity in children born preterm mapped using probabilistic magnetic resonance tractography. Neuroimage 2007;34:896-904.

28. Boardman JP, Craven C, Valappil S, et al. A common neonatal image phenotype predicts adverse neurodevelopmental outcome in children born preterm. Neuroimage 2010;52:409-14.

29. Behrens TE, Johansen-Berg H, Woolrich MW, et al. Non-invasive mapping of connections between human thalamus and cortex using diffusion imaging. Nat Neurosci 2003;6:750-7.
30. Johansen-Berg H, Behrens TE, Sillery E, et al. Functional-anatomical validation and individual variation of diffusion tractography-based segmentation of the human thalamus. Cereb Cortex 2005;15:31-9.

31. Grahn JA, Parkinson JA, Owen AM. The cognitive functions of the caudate nucleus. Prog Neurobiol 2008;86:141-55.

32. Abernethy LJ, Cooke RW, Foulder-Hughes L. Caudate and hippocampal volumes, intelligence, and motor impairment in 7-year-old children who were born preterm. Pediatr Res 2004;55:884-93.

33. Bhutta AT, Cleves MA, Casey PH, Cradock MM, Anand KJ. Cognitive and behavioral outcomes of school-aged children who were born preterm: a meta-analysis. JAMA 2002;288:728-37.

34. Fazzi E, Bova S, Giovenzana A, Signorini S, Uggetti C, Bianchi P. Cognitive visual dysfunctions in preterm children with periventricular leukomalacia. Dev Med Child Neurol 2009;51:974-81.

35. Volpe JJ. The encephalopathy of prematurity-brain injury and impaired brain development inextricably intertwined. Semin Pediatr Neurol 2009;16:167-78.

36. Fukuda S, Yokoi K, Suzuki S, Goto H. Serial ultrasonographic observation of bilateral thalami in low birth weight infants with periventricular leukomalacia. Brain Dev 2011;33:394-9.

37. Song SK, Yoshino J, Le TQ, et al. Demyelination increases radial diffusivity in corpus callosum of mouse brain. Neuroimage 2005;26:132-40.

38. Flodmark O, Roland EH, Hill A, Whitfield MF. Periventricular leukomalacia: radiologic diagnosis. Radiology 1987;162(1 Pt 1):119-24.

39. Zubiaurre-Elorza L, Soria-Pastor S, Junqué C, et al. Magnetic resonance imaging study of cerebral sulci in low-risk preterm children. Int J Dev Neurosci 2009;27:559-65.

40. Wechsler D. Wechsler Intelligence Scale for Children, 4th edn. Madrid, Spain: TEA Ediciones, 2007.

41. Smith SM, Jenkinson M, Woolrich MW, et al. Advances in functional and structural MR image analysis and implementation as FSL. Neuroimage 2004;23:Suppl 1:S208-19.

42. Smith SM. Fast robust automated brain extraction. Hum Brain Mapp 2002;17:143-55.

43. Patenaude B, Smith SM, Kennedy DN, Jenkinson M. A Bayesian model of shape and appearance for subcortical brain segmentation. Neuroimage 2011;56:907-22.

44. Smith SM, Jenkinson M, Johansen-Berg H, et al. Tract-based spatial statistics: voxelwise analysis of multi-subject diffusion data. Neuroimage 2006;31:1487-505.

45. Rothman KJ. No adjustments are needed for multiple comparisons. Epidemiology 1990;1:43-6.

46. Perneger TV. What's wrong with Bonferroni adjustments. BMJ 1998;316: 1236-8.

47. Nichols TE, Holmes AP. Nonparametric permutation tests for functional neuroimaging: a primer with examples. Hum Brain Mapp 2002;15:1-25. 